Vogel treffend wieder. Wegen des vom Maler gelb anstatt schwarz gemalten Schnabels kann sie nicht verworfen werden. Im Journal f. Ornith. 1915 S. 296 habe ich mich weiter darüber ausgesprochen. Jedenfalls wäre es verfehlt, an Stelle eines zweifelhaften Namens einen noch strittigeren zu setzen (Vergl. auch Ibis 1919, S. 253).

Reichenow.

\title{
Die Vögel der Umgebung von Lissa 1./P.
}

\section{Von C. Kayser.}

(Schlufs von Jahrg. 1920 8. 340.)

47. Emberiza hortulana L. Der O $\mathrm{rt}$ o l a $\mathrm{n}$ ist an Alleen und Waldrändern häufig. In den 4 Jahren $1913-1915$ und 1917 war das erste Ankunftsdatum der 30. IV., das letzte der 9. V. und das durchschnittliche der 3. V. Manche singen keine Schlufslaute, sondern nur den ersten Teil der Strophe. Einer hängte noch einen tieferen Schlufston an, sang also in drei Tonlagen. 1917 war er am 22. VII. noch im Gesang, ja sogar am 27. VIII. hörte ich noch einen, allerdings etwas leise, singen.

48. Emberiza citrinella L. Die Gol d a $\mathrm{m} \mathrm{m}$ er ist häufig. Erster Gesang 1914 am 21. II.

49. Emberiza calandra L. Die $\mathrm{Gr}$ a u a $\mathrm{mmer}$ ist ebenfalls häufig. Während ich diese Vogelart in Schlesien nur als Zug- bezw. Strichvogel kennen lernte, ist sie hier auch im Winter anzutreffen. Der hiesige Ausstopfer erhielt Ende Januar 1914 eine Grauammer. Am 9. II. 14. eine Anzahl Grauammern an der Gostyner Chaussee beobachtet. 1913 erster Gesang am 12. II., 1914 am 22. II. Am 12. XI. 13 an der Gostyner Chaussee eine Anzahl beobachtet, welche noch öfters sangen, allerdings nicht so abgerundet, als in der Brutzeit. Am 3. VII. 13 auf dem Felde eine zerrissene Grauammer. Der Gesang ertönt bis tief in den Juli, so am 27. VII. 16 und 27. VII. 17.

50. Passerina nivalis $\mathrm{L}$. Die $\mathrm{S} \mathrm{ch}$ ne e a $\mathrm{mmer}$ wurde 1914 oder 1915 von Herrn Förster Paul in Lasswitz bei Heinrichs. hof im Winter beobachtet.

51. Pyrrhula europaea Vieill. und rubicilla Rchw. Der Gimpel kommt im Winter hier vor. Ob es sich dabei um die gröfsere oder kleinere Form handelt, vermag ich beim Mangel an Belegexemplaren nicht zu entscheiden. Ich notierte am 17. I. und 19. I. 14 auf der Ostpromenade ein $ᄋ$, am 1. II. 14 ebenda 3 o $^{x} \sigma^{x}$ und 1 \%. Sie frafsen dort den Samen von Syringa vulgaris. Am 27. II. 14 ein $O$ in einem Hausgarten. 1915 am 24. I. ein $O$ in einem Hausgarten der Stadt. Am 18. II. 15 4 우 und $1 \sigma^{\top}$ an der Ostpromenade, wo sie die Knospen einer 
Rüster frafsen. Am 11. XII. 16: 2 Stück. - Eine interessante Varietät des Gimpels sah ich lebend vor Jahren bei dem hiesigen Ausstopfer. Es war ein sonst normalfarbiges $\sigma^{x}$ mit etwas mattem Rot. Auf der rechten Seite des Kopfes hatte er vom Schnabel über die Augen bis auf die Kopfmitte einen hellgraurötlichen Streifen, etwa von der Farbe des Bauches eines $Q_{\text {. }}$. Der Vogel war hier im Januar 1912 gefangen und Anfangs ganz normalfarbig.

52. Serinus hortulanus Koch. Der G ir lit z ist hier häufig. In den 5 Jahren 1913 und 1915-1918 war das früheste Ankunftsdatum der 3. IV., das späteste der 3. V. und das durchschnittliche der 15. IV. Auffallender Weise kam der Girlitz in den Jahren 1913 und 1914 in den etwa $48 \mathrm{~km}$ südöstlich von hier gelegenen Trachenberg erheblich eher an als hier. 1913 hörte ich ihn dort schon am 23. III., hier am 3. IV. 1917 sang er noch am 31. VII. Aufser dem gewöhnlichen Lockton, den Naumann mit hitzriki treffend wiebergibt, hörte ich vom Girlitz noch häufig einen einsilbigen wie piep klingenden Lockton, welcher dem des zahmen Kanarienvogels täuschend ähnlich, aber in der mir zugänglichen Literatur nirgends erwähnt ist.

53. Carduelis elegans Rchw. Der Stieglitz ist hier in mäfsiger Zahl vertreten, dürfte auch Brutvogel sein. In den Promenaden, Friedhöfen und gröfseren Gärten. Am 12. XI. 13 frafsen Stieglitze im Kankeler Walde Erlensamen. Am 29. VI. 14 an der Ostpromenade 4 flügge Junge.

54. Chrysomitris spinus L. Der Erlenzeisig kommt hier nur selten vor.

55. Acanthis cannabina L. Der Bluthä $\mathrm{nfling}$ ist hier ziemlich häufig. Einmal - 10. V. 15 - sah ich ihn auch auf dem evang. Friedhof. Auch in den Gärten der Stadt kommt er zuweilen vor.

Nach Acanthis flavirostris L. habe ich bisher immer vergeblich geforscht.

56. Acanthis linaria L. Der Birkenzeisig ist hier seltener Durchzugsvogel. Nach Angabe des Ausstopfers kamen sie hier im Winter 1913/14 zahlreich vor.

57. Chloris hortensis Rchw. Der G rü n li ng ist hier sehr häufig auf Promenaden, Kirchhöfen u. s. w. 1913 erster Gesang am 11. II. Am 9. VI. 13 im Erlenbusch Nest mit 6 Eiern in Manneshöhe. - Im Winter steht an meinem Fenster ein Futterhäuschen, welches 2 enge, runde Eingangslöcher für Meisen hat. An der offenen Rückseite ist zwischen dem Futterhäuschen und der Fensterscheibe ein schmaler Spalt, welcher an den Seiten, um die Sperlinge abzuhalten, mit Pappstreifen verschlossen war. Dessenungeachtet flog ein Pärchen Grünlinge durch den oberen Spalt (zwischen der Decke des Futterhäuschens und der 
Fensterscheibe) aus und ein und stillte seinen Hunger im Innern des Futterhäuschens. - Am 4. VI. 15. Nest mit Jungen auf einem Lebensbaum in Mannshöhe. Der alte Vogel ist beim Füttern aufserordentlich vorsichtig. Am 3. VII. 15 auf der Promenade der Federnkranz eines Grünlings. Im Frühjahr 1916 hörte ich einen Grünling, welcher einen Ruf wie huit, dem Lockton des Phylloscopus rufus sehr ähnlich und ganz verschieden von dem gewöhnlichen Lockpfiff der Grünlinge, hören liefs.

58. Fringilla coelebs L. Der B u chfink ist hier sehr häufig. Manche haben ihren Stand auf Chaussebäumen, fern von Gärten und Wäldern, und nisten auch dort. 1913 den 20. II: 2 Stück gesehen. 1913 den 11. III. erster Schlag auf dem Schlolsplatz, wahrscheinlich von einem hier überwinterten Fink. Am 13. III. war der Finkenschlag allgemeiner und am 21. III. war er auch im Walde zu hören. Am 9. XII. 13 einige Finken auf der Promenade. Mitte Januar 1914 suchten sich Finken ihr Futter auf den Strafsen. 1914 am 22. II. erster Schlag, laut und fleifsig, aber noch nicht abgerundet, im Wolfsruhmer Garten. Man hört in hiesiger Gegend ganz wohlklingende Schläge z. B. eine Art Reitzug mit „Amen“ und den Ausgang Reitjawick. - Am 26. V. 13 brütete ein $\&$ auf 4 Eiern und am 22. V. 13 ein anderes im Kankeler Walde in Augenhöhe ebeufalls auf 4 Eiern. Auffallend bei dieser anscheinend ersten Brut war die Vierzahl des Geleges. Am 7. VI. 14 flügge Junge. Ein $\sigma^{x}$ schlug noch am 6. IX. 13 eifrig, aber unbeholfen, vielleicht ein junger Vogel. - Am 10. IV. und 28. IV. 14 Federkränze zerrissener Buchfinken im Schiestwerderwalde bezw. Reisener Walde.

Im Winter 1914/15 nur e i n überwinternder Fink ơ. 1915 erster Schlag am 22. III. und zwar offenbar von diesem überwinternden Vogel. An diesem Tage waren auch einige zurückgekehrte Finken zu sehen. Am 30. III. 15 waren sie noch in Flügen zusammen. Am 2. IV. 15 sah ich das erste \&. Am 5. IV. 15 erster Finkenschlag i m W a ld e. Am 17. IV. 15 das erste Nest gesehen. Am 13. V. 15 in einem Nest in Augenhöhe 2 Eier. Ein anderes Nest war in reichlicher Mannshöhe in einer noch unbelaubten Astgabel sehr auffallend und weithin sichtbar gebaut. Dieses fand ich später zerstört, wie überhaupt von keiner Vogelart soviel zerstörte Nester zu finden sind, als von dieser, allerdings auch besonders häufigen Art. Am 15. VI. 15 flügge Junge. Letzter Schlag am 14. VII. 15. Im Mai 1915 sah ich mehrere Buchfinken auf einer baumlosen Strafse der Stadt umherlaufen. Im Restaurationsgarten Wolfsruhm sind sie so zahm geworden, dafs einer in eine Semmel hineinpickte, an welcher ich noch die Hand hielt. - 1916 erster Schlag am 12. III. Am 23. VI. 16. ein Nest im Walde kaum $1 \mathrm{~m}$ hoch mit fast flüggen Jungen. Letzter Schlag am 14. VII. 16. Im nächst- 
folgenden Winter am 2. XI. 16 und 16. I. 17 vier überwinternde Buchfinken beobachtet. Im Gymnasialgarten überwinterte wieder ein $\sigma^{x}$, wohl dasselbe wie in früheren Jahren. 1917 erster Schlag am 20. III. - Ein ơ lockte füidpink füidpink, die erste Silbe genau wie Erithacus phoenicurus, Letzter Schlag am 23. VII. und bezw. 26. VII. 1917.

59. Fringilla montifringilla $\mathrm{L}$. Der $\mathrm{B}$ e $\mathrm{r} \mathrm{g}$ in $\mathrm{k}$ ist hier ein seltener Durchzugsvogel. Am 15. XII. $15 \mathrm{sah}$ ich auf einem mit Unkraut bewachsenen Militärreitplatz unter Feldsperlingen, Goldammern und Buchfinken auch 3 Bergfinken. Sie frafsen den Samen von Polygonum aviculare. 1918 sah H. Major Kutter schon am 16. X. einen Bergfink im Reisener Walde.

60. Coccothraustes vulgaris Pall. Der $\mathrm{K}$ e $\mathrm{n} \mathrm{b}$ e i $\int \mathrm{s}$ er ist hier ein spärlicher Brutvogel. Ein Pärchen brütete 1914 in einem kleinen Garten an der Ostpromenade. Das Nest stand auf einem Obstbaum 4-5 m hoch. 1915 beobachtete ich ihn zuerst in den Gärten der Stadt am 17. IV. 1916 zeigten sich die ersten an der Ostpromenade schon am 14. II. Während ich sie sonst im Winter nie beobachtete, sah ich trotz des so strengen Winters am 12. I. 17 einen Kernbeifser in einem Garten an der Ostpromenade.

61. Passer montanus L. Der Feldsperling ist hier so häufig wie ich ihn noch nirgends fand.

62. Passer domesticus L. Der $\mathrm{Hausperling}$ ist hier sehr häufig. Am 5. IV. $15 \mathrm{sah}$ ich auf dem Schlofsplatz ein mit völlig kahlem Oberkopf, wie skalpiert. Die Federn schienen durch Anfliegen an einen Drath oder andern harten Gegenstand abgestofsen $\mathrm{zu}$ sein. Infolge des ungewöhnlich strengen Winters 1916/17 sind mehrere Sperlinge tot gefunden worden und anscheinend viele eingegangen, da man eine Abnahme dieser Vögel bemerkte. Aber leider sah man sie gegen Ende Mai wieder in gröfserer Anzahl. Im Juni nährten sie sie sich ebenso wie die Buchfinken viel von dem am Boden liegenden Rüstersamen.

63. Sturnus vulgaris L. Der $\mathrm{S}$ ta $\mathrm{r}$ ist Brutvogel, aber nur stellenweise, so im Park der Konfirmandenanstalt in Wolfskirch und im Lauber Bruch.

64. Oriolus galbula L. Der P ir o 1 ist in hiesiger Gegend trotz des Wassermangels ein zahlreicher Brutvogel. In der Stadt selbst sind mehrere Paare vorhanden, ferner im Reisener Walde an Stellen mit eingesprengtem Laubholz, im Kankeler Walde, in den Brüchen und in parkartigen Gärten. In den 6 Jahren von 1913-1918 war der früheste Ankunftstermin der 2. V., der späteste der 12 . V. und der durchschnittliche der 6. V. H. Lehrer Parusel in Neuguth beobachtete in gedeckter Stellung bei nur etwa $5 \mathrm{~m}$ Entfernung, wie ein Pirol eime Raupe von Harpyia vinula L. frafs. Im Mai 1913 erhielt der Ausstopfer 
ein an einem Drath verunglücktes Stück. Ein dunkelchromgelbes $\sigma^{\Upsilon}$ hatte neben den gewöhnlichen tiefflötenden Strophen der älteren Männchen auch hoch und dünn klingende Pfiffe, wie man sie sonst von jungen Männchen hört. An Orten, wo viele Menschen verkehren, sind diese Vögel weniger scheu, so z. B. auf dem hiesigen evang. Friedhof. Am 11. V. 15 waren dort 2 hochgelbe Männchen, ferner ein einjähriges und ein zweijähriges (nach der Färbung zu urteilen) zusammen und liefsen fleifsig ihre Pfiffe und den Ruf ,hihähä“ ertönen. Sie hielten sich auf fast unbelaubten Bänmen in mittlerer Baumhöhe und liefsen sich auch durch mehrere sie beobachtende Personen nicht stören. - Am 18. V. 15 brachte das $\sigma^{x}$ des dortigen Brutpaares Niststoff herbei. Am 29. VI. 15 ebenda ein flügges Junges, vom Alten gefüttert. Im Frühjahr 1916 fand ich im Priebischer Bruch ein vorjähriges Nest, welches bis auf ein Loch im Boden noch gut erhalten war. Es stand nur etwa $7 \mathrm{Fufs}$ hoch auf einer jungen Linde dicht am Wege. - Am 25. VI. 16 war schon ein junger Vogel flügge. 1918 hörte ich einen alten Pirol noch am 12. VIII. pfeifen. Wie in anderen Gegenden, so legt auch hier das Volk den ins Gehör fallenden Strophen des Pirols Worte unter und zwar die polnischen Worte: "Zofia wywija" oder mit der hier üblichen Umlautung: „Zofio wywijo" (zu Deutsch: „Sofie tanzt"), eine lautlich in der Tat treffende Wiedergabe der Pirolstrophen. Der Vogel selbst heifst hier wilga oder wywilga. Im Kreise Rosenberg 0./S. spricht das Volk die Pirolstrophen in polnischer Sprache folgendermafsen nach: „Bogu wola Chopu rola - Panu pat" zu deutsch: Gott (gehört) der Wille dem Bauern das Land - dem Herrn der Zins!" Offenbar ist dies ein uralter Spruch aus der Robotzeit. Neuerdings wird von Prof. Voigt und Kleinschmidt die Ansicht vertreten, das Zwitschern des Pirols - nicht die Flötenrufe - sei als sein e i g e n t li c h e r Ges a ng anzusehen. Als Grund für diese Auffassung gibt Voigt an, dafs Vogelkundige diezusammenhängenderen, komplizierten Produktionen als Gesang ansprächen. (Vgl. Verhandlungen des V. Ornith. Kongresses S. 976.) Kleinschmidt bezeichnet in seinem Buch „Singvögel der Heimat" die klangvollen Flötenrufe als Locktöne, dagegen das schwatzende, nicht sehr laute Geplauder als Gesang.

Diese Auffassung ist nach meiner Überzeugung irrig. In früherer Zeit habe ich Pirole in gröfserer Zahl Jahre lang im Käfig unterhalten, worüber ich an anderer Stelle (Ornith. Monatsschrift 1898 S. 331 ff.) berichtet habe. Ich besafs ein Männchen, welches diesen Zwitschergesang niemals hören liefs, obwohl ich es über 3 Jahre besafs und es fleifsig pfiff. Andere Pirole liefsen ihre Flötenstrophen wohl mehr als hundertmal am Tage hören wie im Freien, das Zwitschern aber brachten sie nur selten. Genau so ist es auch im Freien. Ich habe der Beobachtung keiner andern Vogelart soviel Zeit und Interesse gewidmet als der des Pirols, aber das Zwitschern verhältnismälsig selten ge- 
hört, während die Flötenstrophen innerhalb der Gesangszeit bei entsprechendem Wetter stets sehr fleifsig ertönen. Schon hieraus folgt, dafs die Flötenstrophen den eigentlichen Gesang des Pirols bilden, nicht aber jenes balzartige Zwitschern, welches ja auch andere Vögel z. B. Erithacus phoenicurus in ähnlicher Weise neben ihren eigentlichen Gesang hören lassen.

65. Nucifraga caryocatactes $\mathrm{L}$. Der $\mathrm{T}$ a n $\mathrm{n} \in \mathrm{nh}$ äh er ist - hier seltener Durchzugsvogel. Nach Angabe des hiesigen Ausstopfers sind diese Vögel etwa im Jahr 1911 hier aufgetreten. Er zeigte mir ein Belegstück. Wie mir H. Major Kutter hier mitteilt, schofs ein Offizier gegen Mitte Oktober 1917 in einem Garten der hiesigen Vorstadt einen Tannenhäher. Welcher Form die hiesigen Vögel angehörten, vermag ich nicht zu sagen, da ich die Belegstück nicht untersuchen konnte.

66. Garrulus glandarius L. Der E i che $\mathrm{lh}$ äh er ist hier häufig, besonders im Oktober nach eingetretener Reife der Eicheln. Am 10. V. 17 flog ein Flug von 20-30 Stück von West nach 0st. Sie kommen auch in die Friedhöfe der Stadt. Am 16. V. 17 war einer dieser Vögel auf dem evang. Friedhof so dreist, dafs ich ohne Deckung unter dem nicht sehr hohen Baum stehen konnte, auf dem er safs und mich ansah.

67. Pica rustica Scop. Die Elster ist hier ein seltener Brutvogel Am 7. und 10. VIII. 14 kamen 2 Elstern, von denen eine einen normalen, die andere (infolge Mauser?) einen kurzen Schwanz hatte, während der Morgenstunden in die hiesige Stadt und in die Höfe. Da sie ganz auffallend dreist waren und die weifsen Gefiederpartien sehr stark durch Schmutz geschwärzt waren, schienen es Vögel zu sein, die aus der Gefangenschaft entflogen waren. - Am 22. IV. 16 ein Paar bei Fürstenwalde beobachtet. Von dort erbielt ich auch ein Gelege von 7 Eiern.

68. Colaeus monedula spermologus Vieill. Die Do hle ist hier häufig. In frühen Morgenstunden - so am 26. V. 14 um 4 Uhr früh - laufen sie auch auf freien Plätzen der Stadt Futter suchend umher. Sie hatten es anscheinend auf die Reste von Nahrungsmitteln abgesehen, weiche die Besitzer von Schaubuden u. drgl. zurückgelassen hatten. Auch im Winter lassen sie manchmal, ebenso wie Corvus frugilegus, den Spaziergänger nahe herankommen. - Am 20. II. 15 zogen grofse Flüge von Dohlen von Ost nach West. Die hiesigen Dohlen dürften nach einigen Stücken, die ich beim Ausstopfer sah und die in hiesiger Gegend zur Brutzeit geschossen waren, zur F'orm spermologus gehören, zu collaris zeigen sie keine Annäherung.

69. Corvus frugilegus L. Die S a t tk äh e ist hier häufig und hat auffallender Weise in den Kriegsjahren noch an Zahl zugenommen. Am 13. II. 16 kamen sie auf den belebten Platz vor dem hiesigen Bahnhof. Am 8. V. 16 sah ich gegen 30 Saat- 
krähen im Striesewitzer Walde. Der dortige Förster H. Paul sagte mir, dafs die daselbst befindliche Brutkolonie verlassen sei. Im Frühjahr 1918 brütete ein Paar erkwürdigerweise in dem mitten in der hiesigen Stadt belegenen und von Häusern umschlossenen Garten des Hotels Kaiserhof auf einem hohen Baum. Ich selbst sah den Horst und an der Artbestimmung kann kein Zweifel sein, da H. Hotelbesitzer Foest mir den Vogel in charakteristischer Weise beschrieb und H. Major Kutter denselben. auch sah.

70. Corvus cornix L. Die $\mathrm{N}$ e b élk $\mathrm{r}$ äh e ist häufig.

71. Lanius collurio L. Der $\mathrm{r}$ ot $\mathrm{r} \ddot{\mathrm{c}} \mathrm{ckig} \mathrm{e} \mathrm{W} \ddot{\mathrm{u}} \mathrm{r} \mathrm{g} \in \mathrm{r}$ ist hier ebenfalls häufig. 1914 beobachtete ich den ersten am 9. V., 1916 am 13. V. - 1918 sah ich den letzten am 2. IX. Im Erlenbusch befanden sich am 6. VI. 15 in geringer Entfernung von einander 3 Nester dieser Art mit Eiern uud ein leeres Nest. Ein $ᄋ$ hatte schon am 3. VI. 15 ein Gelege von 5 Eiern. Einen flüggen Jungen sah ich am 3. VII. 1917. - H. Lehrer Parusel in Neuguth erzählte mir, dafs er im Jahre 1916 einen jungen Neuntöter in ein Bauer steckte und ihn von den Alten weiterfüttern liefs. Letztere verfütterten merkwürdiger Weise viele Falter von Malacosoma neustria L, aber keine von der damals häufigen Euproctis chrysorrhoea L.

72. Lanius minor $\mathrm{Gm}$. Der $\mathrm{g} \mathrm{r}$ a u e Wü $\mathrm{rger}$ ist hier selten. Ich sah nur zwei ausgestopfte Exemplare aus der Umgegend von Grune.

Vom Vorkommen des Lanius senator L. in hiesiger Gegend erhielt ich noch keine Kenntnis.

73. Lanius excubitor L. Der $\mathrm{Ra} \mathrm{u} \mathrm{bw}$ ü $\mathrm{rg}$ er ist hier selten. Ein ausgestopftes Stück stammt aus der Gegend von Grune. Am 7. IV. 16 ein Exemplar an der Kraschener Chaussee beobachtet.

74. Muscicapa atricapilla L. Der Tra uerfliegen$\mathrm{s} c \mathrm{hnäp}$ per ist hier ziemlich häufig, sein Bestand wechselt aber sehr. In den Promenaden, Gärten und Friedhöfen der Stadt hörte ich in manchen Jahren mehrere (etwa 3), in andern gar keine singenden $\sigma^{\top} \sigma^{\top}$. Im Kankeler Walde kommt er in den Eichenbeständen vor, am häufigsten ist er im Striesewitzer Walde, wo er in Spechtlöchern brütet. Der Gesang endigt bei diesen Vögeln frühzeitig, wohl weil er bei ihnen in noch engererer Verbindung mit dem Fortpflanzungstriebe steht, als bei andern Vogelarten. Voigt sagt zutreffend (Exkursionsbuch 6. Aufl. S. 170): „Ende Mai läfst der Sangeseifer plötzlich nach, und im Juni hört man nur selten noch ein Trauerfliegenschnäpperlied." Ausnahmsweise hörte H. Prof. C. Schulz noch am 21. VI. 14 im Striesewitzer Walde einen singen. 1917 kamen sie erst am 4. V. an, es war überhaupt ein spätes Jahr. 
Am 6. V. sah ich auf dem Durchzug ein ti $\mathrm{efs} \mathrm{chwarz}$ es ð. Merkwürdigerweise zeigen die hier ansässigen Männchen entweder ein graues oder ein nur schwärzliches Kleid. 1917 am 4. IX. den letzten gesehen, 1918 am 13. IX.

Ein Balg eines alten $\sigma^{x}$, aus hiesiger Gegend stammend und zur Zugzeit erbeutet, zeigt auf dem Rücken schwarze Befiederung, an den meisten Stellen mit braunem Anflug. A. infr. mens. $80 \mathrm{~mm}$.

75. Muscicapa grisola L. Der g raue Fliegen s chnäpper ist hier häufig. In den 5 Jahren 1913-1917 war ihr erster Ankunftstag der 28. IV., ihr letzter der 6. V. und ihr durchschnittlicher der 2. V. - 1912 sah ich noch am 15. IX. einen grisola. $1918 \mathrm{zog}$ die grofse Mehrzahl dieser Vögel in der Nacht zum 22. VIII. ab, zurück blieb etwa ein drittel des Bestandes. Die letzten bemerkte ich etwa Mitte September. Diese Vogelart nimmt hier zu. Am 9. VI. 13 schon ein flügger Junger mit ausgewachsenem Schwanz - sehr frühe Brut! Am 23. VI., 3. VII. und 16. VII. 13 ebenfalls flügge Junge, ebenso am 23. VI. 15,14 . VI. 16,17 . VI. 16 und 5. VII. 17 . Es findet offenbar in manchen Fällen eine zweite Brut statt. Am 17. VI. 16 flog ein alter Vogel dem zu fütternden Jungen auf den Rücken und reichte ihm so das Futter. Die flüggen Jungen sind so zutraulich, dafs ich am 24. VII. 17 einen solchen mit der Hand berühron konnte, obwohl er vollkommen flugfähig war. Am 25. VI. 18 konnte ich ebenfalls ein flügges Junges berühren und anfassen.

76. Bombycilla garrula L. Der Seiden s chwanz ist hier ein seltener Durchzugsvogel. Er kam im Januar 1914 hier vor, ich sah ein Stück beim Ausstopfer. Im Winter 1915/16 oder dem darauf folgenden Frühjahr wurde er von H. Förster Paul-Lafswitz beobachtet. Am 8. XI. 16 sah ich einen Flug Seidenschwänze von etwa einem Dutzend. Sie hielten sich längere Zeit in der Nähe der Stadt und in deren Gärten auf.

77. Delichon urbica L. Die Mehlschwalbe ist hier weit weniger zahlreich als die Rauchschwalbe und ist nur in der Vorstadt z. B. bei der Ziegelei neben Wolfsruhm und auf den Dörfern anzutreffen. Am 7. IX 17 sah ich noch eine Anzahl Schwalben über Alt-Laube, die dieser Art anzugehören schienen. Am Nachmittage desselben Tages hatten sich einige Dutzend Rauch- und einige wenige Mehlschwalben auf den Telegraphendrähten über der Roonstrafse hierselbst versammelt und veranstalteten Flugübungen.

78. Clivicola riparia L. Die Uf $\theta \mathrm{rsch}$ wa l be kommt hier in mehreren Kolonien vor. Einige derselben befinden sich in Sandgruben an der Chaussee nach Reisen und fand ich am 17. VII. 18 dort mindestens 2 Kolonien besetzt. Das nächste 
Gewässer, es sind dies der Teich und die Ziegellöcher an der Badeanstalt, sind hiervon etwa $2 \mathrm{~km}$ entfernt. Aufserdew befindet sich, wie mir H. Major Kutter mitteilte, noch eine Kolonie bei Storchnest.

79. Hirundo rustica L. Die $\mathrm{Rauchwalbe}$ ist hier häufig und die eigentliche ,Stadtschwalbe“. In den 4 Jahren 1913-1916 war der erste Ankunftstag der 6. IV., der letzte der 28. IV. und der durchschnittliche der 18. IV. 1914 sah ich die letzten (3 Stück) am 9. X. Am 17. VI. 15 und 17. VII. 16 flügge Junge. 1918 die letzten (6 Stück) am 6. X. und dann noch 3 Stück am 18. X. gesehen.

80. Micropus apus Rchw. Der M a u er segle r ist hier häufig, eine Abnahme nicht $\mathrm{zu}$ bemerken. In den 4 Jahren 1913-1916 war der erste Ankunftstag der 29. IV., der letzte der 4. V. und der durchschnittliche der 1. V. - 1913 sah die ich die letzten am 8. VIII.

81. Caprimulgus europaeus L. Der Z i $\theta \mathrm{g}$ en melker kommt hier nur spärlich vor. Beim Ansitz auf den Birkhahn habe ich ihn in hiesiger Gegend nie gehört. Am 25. IX. 12 flog ein Ziegenmelker über die hiesige Promenade. Im Jahre 1918 wurde nach glaubhafter Mitteilung ebenfalls einer in der Stadt gesehen.

82. Coracias garrulus L. Die B l a u rake kommt hier in mälsiger Zahl vor im Kankeler und besonders im Striesewitzer Walde. Der Schwarzspecht sorgt hier für Bruthöhlen. In jüngster Zeit hat sie sich in dem zuletzt erwähnten Revier verringert, vielleicht infolge der überhand nehmenden Eichhörnchen.

83. Upupa epops L. Der Wi ed eho p f ist nur spärlich vorbanden, und zwar in den Brüchen und im Striesewitzer Walde, hauptsächlich an Orten, wo Vieh geweidet wird.

84. Alcedo ispida L. Der E is v o g e l kommt nur spärlich vor. Der hiesige Ausstopfer erhielt ihn nach seiner Angabe mehrfach aus der Gegend von Priebisch.

85. Picus viridis L. Der $\mathrm{Gr}$ ü $\mathrm{nspecht}$ ist ziemlich häufig. Er besucht auch öfters die städtischen Promenaden und Gärten. Im Oktober 1914 liefs einer auf der Promenade sein „Lachen" erschallen und war so zutraulich, dafs er sich einmal auf einen Baum setzte, von dem ich nur etwa 10 Schritte entfernt war. Selbst ein auf der dicht daneben gelegenen Strafse heranfahrendes Auto veranlafste ihn nicht zum Abfliegen.

86. Dendrocopus minor L. Der Klei n specht wird hier zuweilen, auch in der Stadt, beobachtet. Welcher Form die hiesigen Exemplare angehören, vermag ich mangels eines Belegexemplares nicht zu sagen. 
87. Dendrocopus major L. Der grofs e Buntspecht ist hier ziemlich häufig und auch Brutvogel. Er besucht auch die Gärten und Friedhöfe der Stadt.

88. Dryocopus martius L. Der $\mathrm{S} \mathrm{ch} \mathrm{wa} \mathrm{z} \mathrm{s} \mathrm{pe} \mathrm{cht}$ ist ein ständiger Bewohner des Kankeler und Striesewitzer Waldes.

89. Jynx torquilla L. Der W e n d e tra ls ist hier ziemlich, in manchen Jahren sogar recht häufig. In den 4 Jahren 1914-1917 war der erste Ankunftstag der 16. IV., der letzte der 3. V. und der durchschnittliche der 24. IV.

90. Cuculus canorus $\mathrm{L}$. Der $\mathrm{K} \mathrm{u} \mathrm{c} \mathrm{k} \mathrm{u} \mathrm{c} \mathrm{k}$ ist hier in den Brüchen sehr häufig, sonst spärlich vertreten. 1912 sah ich einen noch am 14. IX. Im Mai 1913 wurden 2 am Draht veruuglückte Kuckucke zum Ausstopfen eingeliefert. Ein am 21. VIII. 14 zum Ausstopfen gebrachter Kuckuck war am Unterleib ganz mit gelbem Fett belegt. Nach Mitteilung des H. Lehrers a. G. Remus flog Mitte Mai 1915 ein roter Kuckuck gegen die Glasscheibe eines hiesigen Gebäudes. Wahrscheinlich war er auf der Nestersuche behufs Eiablage begriffen. Anfangs Juli 1915 erhielt Lenhard einen Kuckuck zum Ausstopfen, bei welchem eine Primärschwinge und auf jeder Seite eine Sekundärschwinge rot - von der Farbe der Turmfalkenfedern - war, während der Vogel sonst normal blaugrau war, - jedenfalls ein jüngeres, in der Umfärbung begriffenes Exemplar.

91. Strix flammea L. Die S ch le i ere u le ist hier selten. Im Frühjahr 1918 wurde ein Paar zum Ausstopfen eingeliefert.

92. Athene noctua Retz. Der Steinkauz war früher hier häufig, hat aber in den letzten Jahren sehr abgenommen. Der Grund liegt wohl darin, dafs er aus abergläubischen Gründen getötet und häufig zum Ausstopfen eingeliefert wird. Ein Mann auf einem benachbarten Dorfe hatte beobachtet, dafs ein bei seinem Gehöft rufender Steinkauz in einer inneren Höhlung des Brunnens nächtigte. Da er eine kranke Frau hatte und in dem Steinkauz den Verkünder des Todes erblickte, zog er denselben Abends aus seiner Höhlung und tötete ihn.

93. Syrnium aluco L. Der W a ldka u z ist häufig, auch im Weichbilde der Stadt. 1914 brütete er auf einem der hiesigen Kirchplätze in einer hohlen Linde. Am 29. V. 15 sah ich durch das Lärmen von Amseln und andern Vögeln aufmerksam gemacht, im dichten Laub einer Linde auf dem reform. Friedhof einen Waldkauz der roten Varietät. H. Lenhard zeigte mir am 9. VII. 15 einen Kohlenmeisenschädel, den er nebst dem ganzen, noch unzerkleinerten Körper der Meise einem toten Waldkauz entnommen hatte.

94. Asio accipitrinus Pall. Die S u m f ohreule wurde einmal Anfangs Oktober 1913 von Priebisch zum Ausstopfen eingeliefert. 
95. Asio otus L. Die W a l d o h reule ist hier Brutvogel und ziemlich häufig. Fräulein Kurzmann, Präparandenlehrerin hier, zog im Frühjahr 1917 eine Waldohreule auf, welche ihr als Dunenjunges aus dem Kankeler Walde gebracht wurde. Der Vogel war gegen die Besitzerin zahm, gegen Fremde, wie ich mich ügerzeugte, recht ängstlich und scheu. Mit dem Kater von Fräulein Kurzmann beäugelte sich die Eule, - alleingelassen wurden die beiden Tiere nicht. Die Eule entflog schliefslich, indem sie sich in der Nacht durch die herabgelassene Fensterjalousie hindurchzwängte. Am 16. VIII. 18 teilte mir Fräulein Kurzmann mit, dafs die ihr entflogene Waldohreule sich noch zeitweise in der Nähe ihres Hauses aufhält. Sie schliefst dies daraus, dafs eine Eule in der Nacht auf dem ihrem Hause gegenüberliegenden Hause sich aufhält, von da Rundflüge macht und gerade so ruft, wie früher die von ihr aufgezogene Waldohreule, nämlich hu hú (der 2. Ton höher, kürzer und betont).

96. Cerchneis tinnunculus L. Der $\mathrm{Tu} \mathrm{rmfalk}$ ist hier häufiger Brutvogel. Ein im hiesigen Tierpark verpflegtes $\sigma^{\Upsilon}$, welches 1913 als Nestling aufgepäppelt wurde, war bis zum Oktober 1915 am Kopf nur an der Backengegend etwas blau, die Schwanzfedern waren doppelfarbig, nämlich in der Mitte blau und an den beiden Seiten rot. Interessant ist diese späte Verfärbung, auf welche allerdings auch die Gefangenschaft von Einflufs sein könnte, obwohl der Vogel recht munter und schön befiedert war und im Freien gehalten wurde. Im März 1915 erhielt der hiesige Ausstopfer einen Turmfalken, welcher die Färbung des ㅇ, aber im Schwanz eine blaue Feder trug, wahrscheinlich ein junges unausgefärbtes $\sigma^{x}$. Das Geschlecht wurde leider nicht untersucht. Im Jahre 1918 hatte H. Lenhard einen Turmfalken, welchen er durch Untersuchung des Eierstockes als \& feststellte und den ich später selbst sah, welcher einen blaugebänderten Bürzel und im Übrigen ein sehr helles Gefieder trug - also ein teilweise hahnenfedriges \$. - Am 17. IX. 1917 sah ich eine Krähe auf einen Turmfalk stofsen, welcher die Flucht ergriff. - Am 4. VII. 18 wurde mir ein junger, noch nicht völlig flügger Turmfalk überbracht.

97. Cerchneis merilla (Gerini). Der Merlinfalk ist hier selten. Ich sah bis jetzt nur ein ausgestopftes Exemplar, erlegt etwa 1914 in hiesiger Umgegend, nach Farbe und Gröfse scheint es 우 zu sein. Es befindet sich in meiner Sammlung.

98. Fabco subbuteo L. Der B a $\mathrm{mfalk}$ ist hier ziemlich häufig und horstet dem Vernehmen nach in den nahen Wäldern nach Guhrau zu. H. Lenhard hatte im Mai 1914 einen lebenden, sehr schönen männlichen Baumfalk im Alterskleide. Derselbe wurde bei Witschenske hiesigen Kreises dnrch einen Schufs in den Flügel leicht verletzt. Nach der Mitteilung des ersten Be- 
sitzers, eines Försters, hat er sich anfangs immer auf den Rücken geworfen und die bei Raubvögeln übliche Abwehrstellung angenommen. Er gab dies indessen bald auf und wurde völlig zahm. Wenn er auf dem für ihn bestimmten Kreuzholz safs, konnte ich ihm ruhig mit dem Finger über den Kopf streichen, er wehrte sich nie und flog auch nicht ab. Den vielen in seiner Nähe befindlichen Käfigvögeln schenkt er keine Beachtung. Selbst wenn er durch ein plötzliches Öffnen der Stubentür aufgescheucht, an einen Vogelkäfig flog und die darin befindlichen Singvögel scheu umherflatterten, nahm der Falke hiervon keine Notiz. Im Gefieder hielt er sich schön und unbestofsen. Mäuse hat er dem Vernehmen nach gern angenommen. H. Lenhard fütterte ihn mit den abgebalgten Körpern kleiner Vögel, von denen er mit Vorliebe die Bruststücke frafs. Besonders fielen seine grofsen Augen auf, mit denen er den $\mathrm{Be}-$ schauer lebhaft und ausdrucksvoll anblickte. Die grofse Zahmheit des Vogels war wohl auch eine Folge seiner Verwundung. Ein zweiter Baumfalk, den H. Lenhard bald darauf erhielt, war schon in früber Jugend in Gefangenschaft gelangt, - benahm sich aber bei Weitem nicht so zahm und gutartig, wie der oben geschilderte Wildfang.

99. Falco peregrinus Tunst. Der W a n d e $\mathrm{f} \mathrm{a} \mathrm{lk}$ e kommt hier zuweilen vor. Am 21. XI. 15 wurde ein Wanderfalk aus Witoslaw zum Ausstopfen eingeliefert, der vorwiegend das Alterskleid, aber noch manche Federn aus dem bräunlichen Jugendkleid trug. Die beiden ersten Handschwingen jedes Flügels waren in der Mauser bezw. im Wachsen begriffen. Gegen Mitte Oktober 1916 wurde wiederum ein Stück zum Ausstopfen eingeliefert, ebenso am 17. I. 1917 von Kankel. Am 14. VII. 1918 sah ich einen toten Wanderfalk, welcher im Walde bei Blotnik etwa $25 \mathrm{~km}$ von hier tot gefunden wurde, nach Färbung und Grölse ein ơ im Jugendkleide. Er war am Kopf stark blutbefleckt und hatte sich offenbar durch Anfliegen verletzt.

100. Falco cherrug Gr. Das einzige Exemplar hiesiger Gegend vom Wü rg falk wurde in der zweiten Hälfte des Oktober 1914 in Górkaduchowna Kreis Schmiegel, etwa $11 \mathrm{~km}$ von hier, erlegt und befindet sich in meiner Sammlung. Es ist ein jüngeres Stück mit blauen Fängen, über welches ich schon Orn. Monatsberichte 1915 S. 43 berichtete. Die Mittelzehe ohne Kralle ist kürzer als der Lauf, Aufsen- und Innenzehe sind gleichlang. Die Färbung entspricht im Wesentlichen der Abbildung im Neuen Naumann Bd. V. Tafel 13. Der Unterleib ist gelblichweifs mit dunklen Schaftstrichen, die kreisförmigen bezw. elliptischen Flecke auf dem Schwanz sind weifslich.

101. Pandion haliaetus $\mathrm{L}$. Den $\mathrm{F}$ is $\mathrm{c} \mathrm{h}$ a d le $\mathrm{r}$ beobachtete ich einmal am 26. VI. 14 am Tharlanger Bruch und aufserdem 
- vielleicht dasselbe Exemplar - vorher am 20. VI. 14 am Domniker See, etwa $20 \mathrm{~km}$ von hier.

102. Haliaetus albicilla L. Vom Se a d le r sind 3 Fälle des Vorkommens bekannt. Am 22. VII. 16 erhielt H. Lenhard einen Seeadler im Alterskleide $\sigma^{\top}$, noch lebend. Derselbe wurde zwischen Blotnik und Grotnik, etwa $22 \mathrm{~km}$ von hier, angeschossen und liefs sich merkwürdiger Weise von dem Schützen im Rucksack gutwillig hierher transportieren. H. Lenhard fafste ihn dann am Kopf an und nickte ihn ab. Er wahr recht wohlgenährt. - Ein zweiter Seeadler im noch dunkelbraunen Gefieder wurde vor 6-8 Jahren bei Storchnest geschossen und steht ausgestopft in der Delikatessenhandlung von Krischker hier. - Ein dritter, ebenfalis dunkelbraun, wurde etwa $1911 \mathrm{im}$ Revier Kraschen durch den H. Hegemeister Grunert daselbst orlegt, in dessen Besitz ich ihn sah.

103. Milvus korschun $\mathrm{Gm}$. Der $\mathrm{schwarze} \mathrm{M}$ ilan brütete nach Angabe des H. Hegemeisters Grunert 1906 oder 1907 im Kraschener Bruch. Im Frühjahr 1917 beobachtete ihn H. Förster Paul-Lalswitz. Ebenso sah ihn H. Forstmeister Dommes im Kraschener Bruch in demselben Jahr.

104. Milvus ictinus Sav. Die $\mathrm{G}$ a bel we ih $\theta$ wurde nur einmal etwa 1910 oder 1911 von H. Förster Paul-Lafswitz beobachtet.

105. Pernis apivorus L. Der W e s pe n b u s a rd wurde einmal - im Mai 1913 - bei Priebisch erlegt und hierher zum Ausstopfen eingeliefert.

106. Archibuteo lagopus Brünn. Der $\mathrm{R}$ a u h f u fs b u s s a r d wird hier öfters erlegt.

107. Buteo vulgaris Leach. Der Mä us $\theta \mathrm{bussard}$ kommt ziemlich häufig vor und ist in den umliegenden Wäldern Brutvogel. Auffallend ist, dafs hier öfters Mäusebussarde mit roströtlicher Färbung zum Ausstopfen eingeliefert werden, wie ich solche in Schlesien nie sah. So am 18. II. 14 ein bei Bojanowo erlegter Bussard, welcher reichliche Mafse zeigte. Seine Oberseite war braun, die Unterseite ebenfalls dunkel, aber stark rostrot angeflogen, die Unterschwanzdeckfedern mit rötlichbraunen (nicht rostroten) Querbinden und ohne breiteres Querband, Bänderung des Schwanzes deutlich. Ende Februar oder Anfangs März 1914 wurde wieder 2 Bussarde derselben Färbung eingeliefert. Den Artkennzeichen von Buteo desertorum Daud. entsprechen diese Vögel nicht, es scheint sich vielmehr um dieselbe Färbungsvarietät $\mathrm{zu}$ handeln, welche schon anderweitig (Journ. f. Ornith. 1871 S. 236) besprochen wurde und welche einen Übergang in der Färbung zwischen Buteo vulgaris und Buteo aesertorum zu bilden scheint. - Im Juni 1915 war im hiesigen Tier- 
park ein jung aufgepäppelter Mäusebussard, der merkwürdiger Weise eine rötlichweifse Unterseite mit Längsflecken hatte, ähnlich einem Hühnerhabicht im Jugendkleide. Es handelt sich dabei wohl um eine nur in der Gefangenschaft vorkommende Farbenvarietät, wie eine ähnliche ja auch beim Wanderfalk in der Gefangenschaft beobachtet wurde. (Hartert, Die Vögel d. paläarkt. Fauna II. S. 1045, Kleinschmidt, Berajah 1912 und 1913).

108. Accipiter nisus L. Der $\mathrm{Sper} b$ e r ist hier ziemlich häufig und nach Mitteilung des H. Hegemeisters Grunert im Kraschener Bruch Brutvogel. Gegen Ende Juni 1915 flog ein Sperber nach einem Sperling in die Stube eines Tischlers in Lasswitz, wurde erschlagen und zum Ausstopfer gebracht. Zwei der mittelsten Schwanzfedern waren ausgefallen und erst in kaum halber Länge nachgewachsen. Es war ein ㅇ. Im Jahre 1918 schofs H. Forstmeister Dommes ein $\sigma^{\star}$, welches soeben eine Singdrossel geschlagen hatte.

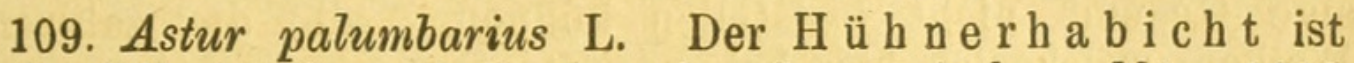
hier ebenfalls häufig und Brutvogel. Gegen Anfang März 1915 erhielt der hiesige Ausstopfer einen jüngeren Hühnerhabicht von Murke hiesigen Kreises. Die Iris war schon rotgelb, obwohl der Vogel noch das Jugendkleid trug, allerdings war die rötliche Unterseite schon stark in's Weifsliche verschossen. Er schien 1913 erbrütet zu sein. Am 12. V. 1916 wurde von Alt-Laube ein Hühnerhabicht $\bigcirc$ mit legereifem Ei eingeliefert, im Eierstock waren noch 4 Eier ausgebildet. - Im Juli 1916 erhielt H. Lehrer Hausdorf einen jungen flüggen Hühnerhabicht, den Landarbeiter bei Friebisch in noch nicht völlig flugfähigem Zustand aufgegriffen hatten. Der Vogel hatte graugrüngelbe Iris, war lebhaft gefärbt, kräftig und so gefrälsig, dafs er Fleisch kröpfte, während wir unmittelbar vor seinem Käfig standen. H. Hausdorf erzählte mir, dafs der Vogel, als die Tür seines Kistenkäfigs einmal einen Augenblick offen stand, ihn, seinen Pfleger, sofort angegriffen und an der Hand verletzt habe. Auf meine Veranlassung kam der Vogel in den zoolog. Garten in Breslau. - H. Hegemeister Grunert erzählte mir, dals or vor längerer Zeit junge Hühnerhabichte in den hiesigen Tierpark brachte. Der eine, in die Voliere gesetzt, zerfleischte sogleich einen dort befindlichen Turmfalk, obwohl H. Grunert ihn vorher zu Hause satt gefüttert hatte. Nach Mitteilung dieses Gewährmannes hat der Hühnerhabicht auch in diesem Jahr - 1918 - bei Tharlang gebrütet.

110. Circus cyaneus L. Die K o rnwei he ist hier häufig und Brutvogel in den hiesigen Brüchen. Ein jüngeres, hier zum Ausstopfen eingeliefertes Stück hatte grüngelbe (nicht, wie man immer in naturgeschichtlichen Werken liest: gelbe) Füfse. Vorn hatten dieselben sogar eine blaue Farbe, vielleicht eine Folge 
einer nach dem Tode eingetretenen Verfärbung. Im Mai 1915 erhielt ich ein altes $\&$, erlegt bei Priebisch. Nach Mitteilung des Ausstopfers hatte es 4 Eier am Eierstock ausgebildet und übrigens im Innern 2 Eidechsen und 1 Frosch. 1916 wuurde das erste Stück zum Ausstopfen, ebenfalls ein $ᄋ$, am 12. II. aus Gurschno hier eingeliefert, welches einen Frosch im Magen hatte. 1918 sah ich das erste Stück, ein altes Weibchen, am 5. III. Über die Schädlichkeit der Kornweihe machte mir H. Hegemeister Grunert interessante Mitteilungen. In dem einen Jahr fand er sehr viele ausgefressene Eier von Fasan, Wildente, Numenius arcuatus u. s. w. Da er Krähen für die Täter bielt, stellte er Eisen mit Hühnereiern als Köder. In diesen Eisen fing er dreimal hinter einander je eine Kornweihe. Von da an lagen keine ausgefressene Eier mehr umher. Trotzdem wäre es schade, wenn die Kornweihen zu stark verfolgt würden, denn der ,weifse Hans", wie sie in Kurland wegen der hellen Farbe der Männchen genannt werden, ist eine grofse Zierde für die Landschaft.

Die Wiesen-, Steppen- und Rohrweihe habe ich für die hiesige Gegend nicht feststellen können. Alle die zahlreichen Weihen, welche ich hier beim Ausstopfer untersuchte und bezw. im Freien mit dem Trïederglase genau erkannte, waren Kornweihen, die nur hier manckmal, weil sie in Brüchen wohnen, von Förstern irrtümlich als "Wiesenweihen" bezeichnet werden.

111. Tetrao tetrix $\mathrm{L}$. Das $\mathrm{B} \mathrm{i} \mathrm{rkhu} \mathrm{h} \mathrm{n}$ ist hier in manchen Revieren häufig, hat aber in den Kriegsjahren sehr abgenommen. Der Grund liegt offenbar teils in Kulturverbältnissen (Grasverpachtung u. s. w.), teils in der Zunahme der Füchse. Am 18. X. 1916 wurde hier ein jüngerer, in de r $\mathrm{Mauser} b$ efindlicher Birkhahn zum Ausstopfen eingeliefert. Am Hals und an den Schultern trug or noch die Federn des Jugendkleides, im Übrigen ein trübes, bräunliches Alterskleid.

112. Coturnix communis Bonn. Die $\mathrm{W}$ a chtel ist hier spärlich vertreten. In der näheren Umgegend ist bisher keine Zunahme zu verzeichnen. Dagegen ist sie bei Feuerstein etwa $17 \mathrm{~km}$ von hier, nach Mitteilung des H. Studienrates Schulz neuerdings häufiger geworden. Mein Gewährsmann führt dies auf den dortigen Anbau von Hirse zurück, da die Wachtel nach seinen 30 jährigen Erfahrungen mit Vorliebe in Hirsefeldern wohnt. Da indessen auch aus andern Gegenden über Zunahme der Wachtel in jüngster Zeit berichtet wird, dürften vielleicht auch noch andere Ursachen für die Zunahme in Frage kommen.

113. Perdix cinerea Lath. Das Rebhuhn ist hier häufig und Brutvogel. H. Studienrat Schulz schofs, wie er mitteilte, im Herbst 1917 ein Rebhuhn, welchen die Schädeldecke vollständig fehlte, so dafs man das Gehirn arbeiten sah. Er nimmt an, dafs die Verletzung durch eine Mähmaschine oder Sense verursacht war. 
114. Turtur communis Selby. Die $\mathrm{T}$ u $\mathrm{rtelta} \mathrm{ub} \theta$ ist hier ziemlich häufig und stellenweise - wio im Priebischer Bruch - auch jedenfalls Brutvogel.

115. Columba palumbus L. Die $\mathrm{R}$ in $\mathrm{ge} \mathrm{lt} \mathrm{a} \mathrm{u} \mathrm{be} \mathrm{ist} \mathrm{hier}$ recht häufig. H. Lehrer Parusel sah bei Neuguth einen Flug von schätzungsweise 75 Stück. Am 9. VI. 15 volles Gelege. Ihr Benehmen ist je nach dem Standort sehr verschieden. Während sie stellenweise - z. B. in Alt-Laube - sehr zutraulich ist und den Beobachter nahe herankommen läfst, flieht sie im Walde den Schützen schon auf weite Entfernung.

116. Columba oenas L. Die $\mathrm{Hohlta} \mathrm{ube}$ ist Brutvogel im Striesewitzer und Kankeler Walde. In ersterem Revier sind mehrere Paare vorbanden und brüten meist in Schwarzspechthöhlen.

117. Phoenicopterus roseus Pall. Der $\mathrm{Fla} \mathrm{ming} 0$ wurde einmal von H. Lehrer Hubrich am 3. V. 14 an dem Wege von Garzyn nach Frankowo hiesigen Kreises in einem Wassertümpel beobachtet. Sein Schnabel war gelblich, an der Spitze schwarz, der Körper des Vogels weifs, der Rücken mit rosigem Anflug, die Schwingen schwarz, die Ständer rot. Nach dieser von H. Hubrich gegebenen Beschreibung handelt es sich um ein jüngeres, aber nicht mehr im Jugendkleid befindliches Exemplar. Der Vogel liefs sich auf etwa $30 \mathrm{~m}$ nahe kommen und flog dann im Halbkreise an das andere Ufer des Tümpels. Das Tier benahm sich weder scheu, noch zeigte es Zeichen von grofser Ermattung. Auf einen Kreischlaut, wie ihn etwa Wildenten beim Hochgehen hören lassen, hob der Vogel aufhorchend den Kopf und antwortete mit einem schnarrenden Gekrächze. Gegen 5 Uhr war er verschwunden.

Nach einer Meldung der „Breslauer Zeitung" vom 7. VI. 14 schofs Graf Rothkirch aus Bärsdorf-Trach bei Haynau einen Flamingo. Es scheint dies dasselbe Exemplar zu sein, welches H. Hubrich beobachtete. Es dürfte sich um einen der Gefangenschaft entflogenen Flamingo handeln, wofür schon die Tatsache spricht, dafs der Vogel die dieser Art eigentümliche Scheu vor den Menschen gänzlich vermissen liefs. Nachträglich in Görlitz eingegangene Nachrichten lassen ja auch darauf schliefsen, dafs der am 17. X. 05 in der Görlitzer Heide erlegte Flamingo aus dem zoologischen Garten in Hannover entflogen war.

118. Ardea cinerea L. Der $\mathrm{F}$ is ch reiher kommt hier öfters an den Landgräben vor. Brutvogel ist or nur in einer etwa $18 \mathrm{~km}$ entfernten Kolonie im Luschwitzer Forst. Der Reiherstand befindet sich dort im gut belaubten Eichenhochwald mit einigen eingesprengten Kiefern. Es sind über 40 Horste vorhanden, von denen allerdings der gröfste Teil unbewohnt ist. Sie stehen fast sämtlich auf Eichen, bis 8 Stück auf einem und 
demselben Baum, - nur einer befindet sich auf einer Kiefer. $\mathrm{Am}$ 21. V. 15 flügge Junge. - Junge Fischreiher wurden in dem hiesigen Tierpark eingeliefert, wo man sie an einem Flügel koupierte, wie man dies bei Enten zu tun pflegt. Infolgedessen brachen sie aber den einen Ständer.

119. Ardetta minuta L. Die $\mathrm{Z}$ we rgrohrdom mel wurde nur einmal - im Mai 1916 - bei Retschke erlegt.

120. Ciconia ciconia L. Der weifse Sto r ch ist vereinzelter Brutvogel, so in Priebisch und Kraschen. Auf dem Durchzug ist er manchmal sehr häufig. So beobachtete H. Forstmeister Dommes Anfangs August 1916 bei Kraschen Hunderte von Störchen. Am 1. VI. 15 in Priebisch fast flügge Junge. Die Schnäbel der letzteren sind immer schwarz. Einem der zahmen Störche im hiesigen Tierpark näherte sich mehrmals spielend eine junge, halbwüchsige Ziege mit stofsbereitem Kopf. Der Storch hackte sie jedesmal bei der Annäherung mit dem Schnabel fest auf den Kopf, zuletzt verfolgte er fauchend die Fliehende.

121. Ciconia nigra L. H. Forstmeister Dommes teilte mir mit, dafs er im Frühjahr 1917 am Kraschener Bruch einen $\mathrm{schwarz}$ en Stor ch sah und ebenso einmal - wohl dasselbe Exemplar - auf den Fürstenwalder Wiesen. Auch erhielt H. Lenhard im August 1915 ein Stück im Jugendkleide von Górka duchowna.

122. Fulica atra L, Das B $\mathrm{l}$ ä $\mathrm{fs} \mathrm{h} u \mathrm{hn}$ ist hier ein nicht seltener Brutvogel auch an kleinen Tümpeln, besonders zahlreich ist es in Lindensee. Im Frühjahr 1917 erhielt der Ausstopfer ein überwintertes Bläfshuhn.

123. Gallinula chloropus L. Das g r ü n $\mathrm{f} u ̈ f \mathrm{sig}$ e T e i c h$\mathrm{huhn}$ ist weniger zahlreich als das vorhergehende, aber auch Brutvogel.

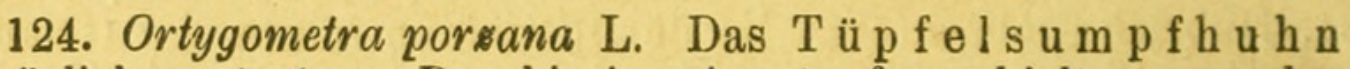
ist spärlich vertreten. Der hiesige Ausstopfer erhielt es aus der Gegend von Grune.

125. Crex pratensis Bchst. Der Wa c h t el k ö nig kommt ziemlich spärlich im Priebischer, Tharlanger und Kraschener Bruch usw. vor, ist auch Brutvogel.

126. Rallus aquaticus L. Die Wasserralle wurde wiederholt hier zum Ausstopfen eingeliefert, so gegen Ende Januar 1914 aus der Gegend von Leiperode, ferner gegen Anfang April 1914.

127. Grus communis Bchst. Der $\mathrm{K} \mathrm{ran} \mathrm{i} \mathrm{ch}$ ist hier Brutvogel in verschiedenen Revieren. Im Lauber Bruch sollen früher 2 Brutpare gewesen sein, sio scheinen aber abgenommen zu 
haben. Im Kraschener Bruch brüten nach Annahme des H. Hegemeisters Grunert ebenfalls 2 Paar. Auch im Tharlanger und Priebischer Bruch ist der Kranich öfters zn sehen.

128. Otis tarda L. Die $\mathrm{g} \mathrm{r}$ of $\mathrm{se} \mathrm{Tr}$ a $\mathrm{p} \mathrm{pe}$ ist ebenfalls Brutvogel, mindestens in einem Paar bei Garthe. Aufserhalb der Brutzeit sieht man Flüg $\theta$ von $30-40$ Stück. In strengen Wintern kommen sie bis an das Dorf Lasswitz heran. Dominialäcker ziehen sie der grölseren Ruhe wegen den Bauernäckern vor. Am 8. VII. 16 sah ich vom Eisenbahnwagen aus unweit Striesewitz 2 oder 3 Trappen und dann an demselben Tage bei Garthe 2 alte und 3 junge Trappen, letztere stärker als Haushühner. Der dortige Vogt sagte, es seien sonst 5 alte Trappen dort. Ihr Flug ist langsam und mit flachen Flügelschlägen, ihr Flugbild mit dem starken ausgestreckten Hals erinnert sehr an Anser ferus. Mit dem Trïeder konnte ich genau sehen, dals sie im Fluge die Ständer nach vorn, lose an den Körper gezogen, halten. Danach ist die Angabe Naumann's von dem Nachhintenstrecken irrig, dagegen die Darstellung in Brehm's Tierleben 4. Aufl. Bd. VII. S. 203 zutreffend.

129. Scolopax rusticola L. Die W a lds c h n $\theta$ p f e kommt bier in mäfsiger Zahl vor. Wie mir H. Forstmeister Dommes mitteilte, war sie im Jahre 1918 häufiger, er hat sie auch z. B. am 16. V. 18 beobachtet und nimmt an, dafs sie im Reisener Revier brütet.

130. Gallinago coelestis Frenz. Die B ekas s in e kommt hier gleichfalls in mäfsiger Zahl vor - z. B. im Priebischer Bruch. Am 22. X. 16 traf ich ein Stück an einem Lehmausstich hinter Wolfsruhm.

131. Numenius arquatus L. Der g rofs e $\mathrm{Brachvogel}$ ist hier Brutvogel und ziemlich häufig, so am Priebischer Bruch, Kraschener Bruch, auf den Wiesen bei Fürstenwalde u. s. w. In der Nähe ihrer Nester fliegen sie viel umher, pfeifen laut und häufig und greifen nach Art der Kiebitze andere Vögel an. Sie pfeifen einsilbig tüt, zweisilbig tui (der Ton auf der letzten Silbe) und manchmal auch dreisilbig. Aufserdem lassen sie ein Trillern wie lülülülülü hören. Einer dieser Vögel griff einen vorüberstreichenden Kranich an, und dieser öffnete, wie ich durch das Fernglas erkennen konnte, zur Abwehr etwas den Schnabel. Im März 1917 wurde ein Exemplar bei Reisen tot aufgefunden, wohl ein Opfer des strengen Winters. - Manchmal fliegt dieser Vogel mit ziemlich schnellem Flügelschlag und senkt sich dann im Gleitflug, um sodann wieder von Neuem mit diesem Flugspiel zu beginnen.

132. Limosa aegocephala L. Die Uf e r s c h n e p f e wurde nach Mitteilung des H. Lehrers a. G. Remus einmal im Priebischer Bruch geschossen. 
133. Tringoides hypoleucos L. Vom Flu fs uf erlä ufer sind nur zwei Fälle des Vorkommens bekannt geworden. Im Juni 1914 verunglückte bei Deutsch-Wilke ein Exemplar im Jugendkleide an einem Draht und kam in meine Sammlung. Am 28. IV. 16 wurde ein zweites Stück aus Woynowitz zum Ausstopfen eingeliefert.

134. Totanus fuscus L. H. Lenhard erhielt einen d un klen Wasserlä ufer Anfangs Oktober 1914. Er war in hiesiger Gegend tot aufgefunden.

135. Totanus calidris L. Der $\mathrm{R} 0 \mathrm{t} \mathrm{s} \mathrm{h}$ e $\mathrm{nk}$ el kommt auf den Wiesen bei Priebisch und Kraschen als Brutvogel vor.

136. Totanus littoreus L. Vom he 11 e n W a s s erläu f er wurde ein Exemplar Ende August 1915 bei Gurschno erlegt.

137. Oedicnemus scolopax $\mathrm{Gm}$. Der $\mathrm{T}$ ri $\mathrm{\theta l}$ ist hier ziemlich häufig an Kiefernfeldhölzern auf sandigem Boden. Auch traf ich ihn wiederholt auf einem Schlage im Reisener Walde, auf welchem die jungen Kiefern etwa 1 Fufs hoch und ziemlich lückenhaft stehen. Dieser Schlag ist vom Waldrand über $500 \mathrm{~m}$ entfernt.

138. Vanellus capella Schäff. Der Kiebitz ist hier ziemlich häufig am Priebischer Bruch, bei Neuguth, Grume u. s. w.

139. Charadrius apricarius L. H. Lenhard, welcher einen G oldregen pfejfer aus Kuttlau in Schlesien erhielt, versicherte mich, diese Art auch aus hiesiger Gegend schon erhalten $\mathrm{zu}$ haben.

140. Cygnus olor Gm. Im Jahre 1917 schofs ein hiesiger Offizier auf einen wilden Schwan im Tharlanger Bruch. Die Art steht leider nicht fest, aber im Februar 1917 wurden zwei H ö ckers chwäne bei Zedlitz unweit Fraustadt erlegt, von denen ich einen selbst sah, und in demselben Monat wurden nach Mitteilung von H. Major Kutter bei Oberleschen Kreis Sprottau mehrere Wildschwäne erlegt, welche teils als $C$. olor, teils als musicus bestimmt wurden und von denen er selbst einen olor schofs.

Im halbzahmen Zustande lebt $C$. olor auf dem hiesigen Schlofsteich. Sie sorgen durch Verzehren von Schmarotzerpflanzen für die Klarheit des Wassers. Während sie sich früher fortpllanzten, haben sie von $1914 \mathrm{ab}$ infolge des Kriegsfutters sich zwar gepaart, aber nicht mehr Eier gelegt, leiden auch an einer abnorm langen Mauser. Der Singschwan Cygnus musicus ist hier in der näheren Umgegend noch nicht vorgekommen, wohl aber im März 1914 bei Blotnik etwa $24 \mathrm{~km}$ von hier, von wo H. Lenhard 2 geschossene Exemplare erhielt. Dem Vernehmen nach wurden 15 (!) Stück erlegt. Man sieht hieraus, wie wenig den berechtigten Auforderungen des Naturschutzes Rechnung getragen wird. 
141. Anser ferus Brünn. Die Graugans ist nach Angabe des H. Hegemeisters Grunert im Kraschener Bruch in einem Paar Brutvogel.

142. Anas crecca L. Die $\mathrm{Krickente}$ brütet ebenfalls nach demselben Gewährsmann im Kraschener Bruch.

\section{Anas querquedula L. Die $\mathrm{Kn}$ ä $\mathrm{ck} \theta \mathrm{n} \mathrm{t} \theta$, sowie}

144. Anas acuta L. Die S p i e $\int \mathrm{s}$ e n t $\theta$ und

145. Anas penelope L. Die $\mathrm{P}$ feif e $\mathrm{n}$ te sind nach Mitteilung des H. Hegemeisters Grunert im Kraschener Bruch schon vorgekommen.

Ein im hiesigen Tierpark gehaltener Erpel der Spiefsente gedeiht seit 9 Jahren tadellos und vermausert auch trotz des Kriegsfutters gut und vollständig.

146. Anas boschas L. Die S to ckente brütet im Priebischer und Kraschener Bruch und anderwärts. Ferner befinden sich halbzahme, freifliegende Stockenten auf dem hiesigen Schlofsteich, wo sie bis vor Jahresfrist auch brüteten. Am 8. V. 13 sah ich dort ein Junges von Wachtelgröfse. Die andern Jungen sind nach Aussage des Wärters durch das kalte Wetter zu Grunde gegangen. Die Jungen nähren sich viel von den über dem Wasserspiegel fliegenden Insekten, nach welchen sie schon in frühester Jugend mit grölster Schnelligkeit und Gewandtheit schnappen. Eine Mückenplage war am Schlofsteich noch nie zu bemerken, wie man ja auch in andern Städten halbzahme Stockenten zur Bekämpfung der Mückenplage mit bestem Erfolg angesiedelt hat. Da die nähere Umgebung der Stadt wasserarm ist, gewährt es einen um so reizvolleren Anblick, wenn ein Flug dieser Enten über die Häuser und Strafsen hinwegstreicht. 1914 hatten 2 Erpel schon am 15. IX. das Prachtkleid angelegt, während die andern noch das Sommerkleid trugen. Am 30. V. 15 flogen 2 Entenmütter, welche kleine Junge führten, auf einen Schwan los und schlugen denselben in die Flucht. Die Erpel überlassen die Führung der Jungen ganz den Müttern. Ein Paar begattete sich am 27. X. 15 bei $-1^{0} \mathrm{R}$. Ebenso beobachtete ich eine Begattung am 1. I. 17 nach mehrfachem gegenseitigen "Antrinken" im Wasser. Das $\sigma^{x}$ streckte darauf den Kopf vorn gerade über das Wasser und schwamm um das $\&$ herum. Bei der Begattung hörte ich keinen Laut. - Bei den Jungen liefs das $\&$ ein leises, unterdrücktes „wag“ hören, das nur in der Nähe hörbar ist.

147. Spatula clypeata L. Die L öffelente ist nach Mitteilung des H. Hegemeisters Grunert im Kraschener Bruch Brutvogel.

148. Fuligula clangula L. Die Schellente ist nach demselben Gewährsmann schon im Kraschener Bruch vorgekommen. 
149. Fuligula nyroca Güld. Die Mo o r e n te wurde in einem Exemplar im August 1914 aus Reisen zum Ausstopfen hier eingeliefert.

150. Fuligula cristata Leach. Im März 1915 wurden 2 Stück Reiherenten in Grätz bei Storchnest erlegt, die ich beim Ausstopfer sah.

151. Hydrochelidon nigra L. Die $\mathrm{Tr}$ a u e $\mathrm{rschw}$ al be ist nach Angabe des H. Lenhard nicht selten bei Lindensee. Ich sah bei ihm ein Belegstück.

Die Flufsseeschwalbe Sterna hirundo L. ist, soweit mir bekannt, in der näheren Umgebung noch nicht vorgekommen. Ich sah nur einmal - 20. VI. 14 - ein Exemplar am Domniker See, etwa $20 \mathrm{~km}$ von hier.

152. Rissa tridactyla L. Eine dreizehige Möwe wurde am 18. II. 16 hier $\mathrm{znm}$ Ausstopfen eingeliefert. Es war ein 1915 erbrüteter Vogel. Ein Mann hatte ihn bei Schwetzkau tot auf dem Felde gefunden. Es war jedenfalls 'dieselbe Möwe, welche ich am 7. XII. 15 überwinternd in Wolfskirch - also $4 \mathrm{~km}$ von Schwetzkau - an einem kleinem, von Hausenten bewohnten Teich antraf, der in nächster Nähe des Weges und der Gebäude liegt. Die Möwe war ziemlich dreist und flog aufgescheucht über dem Dorf umher, kehrte aber bald zu dem Teich wieder zurück.

153. Larus ridibundus L. Die $\mathrm{L}$ a $\mathrm{ch} \mathrm{m}$ ö $\mathrm{w} \theta \mathrm{kommt}$ hier nicht selten vor. 1914 sah ich die ersten am 15. III.

154. Larus fuscus L. Von der $\mathrm{Her}$ ing $\mathrm{sm} \ddot{\text { w }} \mathrm{e}$ wurde ein Stück im Alterskleide aus Retschke am 8. X. 15 zum Ausstopfen hierher eingesandt. Iris gelb.

155. Stercorarius parasiticus L. Eine S chmarotzerra ubmöwe wurde 1912 bei Retschke erlegt. Ich sah sie ausgestopft.

156. Colymbus nigricans Scop. Der $\mathrm{Z}$ wer $\mathrm{g} \mathrm{s}$ t e if $\mathrm{s} f \mathrm{u}$ Is ist, wie mir H. Hegemeister Grunert mitteilte, vorübergehend im Kraschener Bruch. vorgekommen.

157. Colymbus nigricollis Brehm. Am 25. IV. 15 sah ich beim Ausstopfer ein Par Schwarzhalsst $\theta$ i $\int \mathrm{sfü}\lceil\mathrm{s} \theta$, von Klein-Kreutsch eingeliefert. Iris blutrot.

158. Colymbus cristatus L. Der $\mathrm{Haub}$ ensteif $\mathrm{s} f \mathrm{u} \int \mathrm{s}$ kommt auf den hiesigen Seen - Klein-Kreutscher, Storchnester See u.s.w. - ziemlich häufig vor. Ende Februar 1915 erhielt H. Lenhard aus der Gegend von Murke einen Haubensteifsfufs, der sich dort schon längere Zeit herumgetrieben haben soll. Die Spitze des Oberschnabels fehlte, wohl infolge eines Schusses. 
159. Urinator arcticus $\mathrm{L}$. Vom $\mathrm{P}$ o la $\mathrm{rt}$ a $\mathrm{u} \mathrm{ch}$ e $\mathrm{r}$ wurden im November 19153 Stück im Jugendkleide bei Retschke erlegt und zum Ausstopfen eingeliefert. -

Zum Schlufs will ich noch einer interessanten Beobachtung Erwähnung tun, welche vor langen Jahren hier gemacht wurde und von welcher mir die H. Rechnungsräte Gimmler und Wiczienski Mitteilung machten. Ein junger Vogel, welcher anscheinend in einem Tümpel in den hiesigen Bahnhofsanlagen gebadet hatte und $\mathrm{zu}$ tief in das Wasser geraten war, wurde von einem grofsen, g r ü nen W a s e rfros ch erfafst, welcher ihn in die Tiefe zu ziehen suchte. Als H. Rechnungsrat Wiczinski den Vogel herauszog, hing der Frosch noch an ihm er hatte dem Vogel an der Seite gepackt. Der Vogel, welcher alsbald tot war, soll ein junger Insektenfresser, Nachtigall oder Grasmücke, gewesen sein. Über ähnliche Fälle berichten Lenz, Naturgeschichte Bd. III. S. 60 und Prof. Hennicke, Handbuch des Vogelschutzes S. 90.

\section{Zur Frage der Mlimikry der Kuckuckseier.}

\section{Von Friedrioh von Lucanus.}

Mit dem Brutparasitismus des europäischen Kuckucks steht die Frage nach der Anpassungserscheinung der Eier im engsten Zusammenhang. Sie wurde von den älteren Autoren teils bejaht, teils verneint, oder auch als „unentschieden" offengelassen, und auch heute sind die Meinungsverschiedenheiten der Ornithologen und Oologen noch immer geteilt. Unter den älteren Forschern treten $\mathrm{K} \mathrm{unz}, \mathrm{Glog}$ er und $\mathrm{B}$ a l d a $\mathrm{m} \mathrm{u} s$ voll und ganz für die Anpassungstheorie ein, indem die beiden Erstgenannten sogar soweit gehen, dafs sie dem Kuckucksweibchen die Fähigkeit zuschreiben, sein Ei der jeweiligen Färbung der Eier desjenigen Vogelpaares, in dessen Nest es gerade legt, anzupassen, während $\mathrm{B}$ a l d a m u s meint, dafs dasselbe Kuckucksweibchen stets gleichgefärbte Eier legt, die in ihrer Farbe den Eiern der Stiefmutter ähneln, und dafs die Anpassung dadurch zu Stande kommt, dafs das Kuckucksweibchen in der Regel nur die Nester derjenigen Vogelart wählt, von der es erzogen wurde.

Im Gegensatz hierzu verwerfen $\mathrm{K}$ ow ley, L a n do is, Rey und W alter die Anpassungstheorie vollständig. So behauptet R e y in seinem "Haushalt des Kuckucks", dafs unter den von ihm aufgefundenen Kuckuckseiern nur $9 \%$ eine Übereinstimmung mit den Nesteiern zeigten, hingegen $91 \%$ in Färbung und Zeichnung völlig abwichen, und W alter berichtet, dafs von den bis zum Jahre 1888 von ihm gesammelten 250 Kuckuckseiern noch nicht ein Dutzend den Nesteiern ähnlich war. Noch weiter gehen die Gebrüder M üller mit der Behauptung, dafs 


\section{$2 \mathrm{BHL}$ \\ Biodiversity Heritage Library}

Kayser, Charles. 1921. "Die Vögel der Umgebung von Lissa i./P." Journal $f u$

r Ornithologie 69, 218-239. https://doi.org/10.1007/bf02537628.

View This Item Online: $\underline{\text { https://www.biodiversitylibrary.org/item/43096 }}$

DOI: https://doi.org/10.1007/bf02537628

Permalink: https://www.biodiversitylibrary.org/partpdf/141136

\section{Holding Institution}

MBLWHOI Library

\section{Sponsored by}

MBLWHOI Library

\section{Copyright \& Reuse}

Copyright Status: No known copyright restrictions as determined by scanning institution.

This document was created from content at the Biodiversity Heritage Library, the world's largest open access digital library for biodiversity literature and archives. Visit BHL at https://www.biodiversitylibrary.org. 